\title{
Rumfitt on Truth-Grounds, Negation, and Vagueness
}

\author{
Richard Zach
}

the date of receipt and acceptance should be inserted later

Classical logic is characterized by the familiar truth-value semantics, in which an interpretation assigns one of two truth values to any propositional letter in the language (in the propositional case), and a function from a power of the domain to the set of truth values in the predicate case. ${ }^{1}$ Truth values of composite sentence are assigned on the basis of the familiar truth functions. This abstract semantics immediately yields an applied semantics in the sense that the truth value of an interpreted sentence is given by the truth value of that sentence in an interpretation in which the propositional variables are given the truth values of the statements that interpret them. So if $p$ is interpreted as the statement "Paris is in France" and $q$ as "London is in Italy" then the truth value of " $p \vee q$ " is $|p \vee q|$ where the interpretation $\mid$ is given by $|p|=T$ and $|q|=F$. And since the truth value of $|A \vee B|$ is defined as

$$
|A \vee B|= \begin{cases}T & \text { if }|A|=T \text { or }|B|=T \\ F & \text { otherwise }\end{cases}
$$

we have that $|p \vee q|=T$, and so that "Paris is in France or London is in Italy" is true.

On the basis of this semantics, we can, as is done in any introductory logic textbook, define an implication relation: if $X$ is a set of sentences, then $X \Rightarrow A$ if, for every interpretation || such that $|B|=T$ for all $B \in X$, also $|A|=T$. This formal entailment relation can likewise be used to define a logical entailment relation on statements. A statement $A$ entails a statement $B$ iff $A^{\prime} \Rightarrow B^{\prime}$, where $A^{\prime}$ and $B^{\prime}$ are correct symbolizations of the statements $A$ and $B$, respectively.

The question of whether classical logic is the correct logic is the question of whether the implication relation so defined agrees with the pre-theoretic notion of implication between statements. Typically, and reasonably, we gloss over the intermediate step of symbolizing statements in English into the formal language

Richard Zach

University of Calgary, Department of Philosophy, 2500 University Dr NW, Calgary, AB T2N0A9, Canada, http://richardzach.org/, E-mail: rzach@ucalgary.ca

1 That is, a one-place predicate symbol is assigned a function from $D$ to $\{T, F\}$, a two-place predicate a function from $D^{2}$, etc. 
of propositional logic, and do not distinguish between the classical implication relation between (English) statements and the implication relation between the sentences that symbolize them. Whether or not it is admissible, always or in a specific case, to symbolize a statement in one way or the other, may of course have a bearing on the question. Let us set this aside, and suppose, e.g., that if $p$ and $q$ are as above, $p \vee q$ symbolizes "Paris is in France or London is in Italy," $\neg p$ symbolizes "Paris is not in France," etc.

One strategy to argue for the thesis that classical logic is correct would be to argue for the thesis that classical two-valued semantics faithfully captures the intuitive semantics of truth and falsehood. That intuitive semantics relies on the thesis that every basic statement is either true or false (and not both) at every appropriate context of evaluation, and sentences formed by negation, conjunction, disjunction are true or false (at a context) iff their composite sentences exhibit the patterns of truth values specified by the classical truth functions (at a context). Both of these together of course entail bivalence, the claim that every sentence is true or false. This strategy faces the difficulty that there are many cases of statements for which it is doubtful that every context of evaluation does determine one and only one of truth or falsity as their truth value. Basic statements involving vague predicates, such as "Mary is rich" where Mary is a borderline case of a rich person, are perhaps the most pedestrian examples. Challengers to classical logic have suggested other examples of statements claimed to neither be true nor false. Dialetheists suggest that some statements are both true and false, and so also reject one crucial assumption of the above argument.

However, the argument outlined above is not the only possible argument to justify classical logic. For two-valued semantics is not the only semantics that characterizes the classical entailment relation. Classical logic could be supported by giving a parallel argument in which the role of truth values is played by some other entities, and the connective were interpreted by functions of these other entities, so long as that new semantics characterizes the same classical implication relation. That is Rumfitt's strategy in his Boundary Stones of Thought (Rumfitt 2015).

\section{Pretopologies}

The deviant semantics for classical logic that serves this purpose is the pretopology semantics of Sambin (1995). It will be useful to review that semantics: Rumfitt eases his readers into accepting that semantics bit by bit, with a precursor introduced in Chapter 5, a simplified semantics leaving out negation in Chapter 6, and a more complicated semantics in Chapter 7, including negation. So there is unfortunately no one place to go to that discusses all aspects of the proposed semantics. The appeal to Sambin's work is crucial, but the definitions are not the same, so one might wonder how Sambin's results apply.

First, let's review Sambin's semantics. A pretopology is a set $S$ with a combination operator $\bullet$ on $S$ and a $\bullet$-compatible closure operator on $\wp(S)$, i.e., a function 
$C: \wp(S) \rightarrow \wp(S)$ that satisfies

$$
\begin{aligned}
& C(U) \bullet C(V) \subseteq C(U \bullet V) \\
& U \subseteq C(U) \\
& U \subseteq V \Rightarrow C(U) \subseteq C(V) \\
& C(C(U)) \subseteq C(U)
\end{aligned}
$$

where $U \bullet V=\{a \bullet b: a \in U, b \in V\}$. The operator $\bullet$ is associative and has a unit 1 . Sambin gives an equivalent definition in terms of a precover $\triangleleft$; $a \triangleleft U$ iff $a \in C(U) .^{2}$

Rumfitt appeals to Sambin's completeness results in a number of places, and so it may also be useful to trace the relationship between Sambin's semantics and Rumfitt's. Pretopologies provide a sound and complete semantics for a range of logics, of which some are ruled out by the required soundness of a number of some rules of inference uncontested by Rumfitt's imagined interlocutors. Weakening requires that for all $x \in S, x \in C(1)$, i.e., $C(1)=S$. Contraction requires that $x \in C(x \bullet x)$. And explosion requires that contradiction $(\perp)$ is evaluated as $C(\emptyset)$.

The crucial link between Rumfitt's truth-ground semantics and pretopologies is the notion of a possibility, which are the truth-grounds of statements. Possibilities are like possible worlds or states of affairs, but they need not determine the truth value of every atomic statement. E.g., the possibility that $a$ is red determines the truth value of " $a$ is red," but not of " $a$ is large." Rumfitt defines the closure $C(U)$ of a set of possibilities $U$ as the set of all possibilities which make true all statements made true by every possibility in $U$ (p. 162). Any set of possibilities of the form $C(U)$ (i.e., where $U=C l(U)$ ) is called closed. $^{3}$ Possibilities are partially ordered by the relation of determination: $y$ determines $x, x \leq y$, iff every statement true at $x$ is also true at $y$ (p. 166). E.g., the possibility that $a$ is crimson determines the possibility that $a$ is red. From the definitions we have that $x \leq y$ iff $x \in C(y)$. Assuming that no two distinct possibilities can make the same statements true, $\leq$ is anti-symmetric. Possibilities can be combined: the combination $x \bullet y$ of $x$ and $y$ is the least (in the partial order $\leq$ ) possibility $z$ which determines both $x$ and $y$. Combination is compatible with closure: $C(U) \bullet C(V) \subseteq C(U \bullet V)$. There is a least determinate possibility $T$, which makes true those statements that are true at every possibility. The least possibility that determines both $x$ and $\mathrm{T}$ is just $x$ itself, since every statement true at 1 is also true at $x$. So $x \bullet \top=\top \bullet x=x$. We also assume that there is a most determinate possibility $\perp$, which makes every statement (including contradictions) true (p. 188). This, Rumfitt acknowledges, is an idealization, a mere "bookkeeping device."

If we accept this picture, we can establish that possibilities form a pre-topology: the set of possibilities is $S$, combination of possibilities is the $\bullet$ operator, $T$ is the unit 1 of $\bullet$, and $C$ is a closure operator that satisfies Sambin's conditions. The closure $C(\mathrm{~T})$ of the least determinate possibility is $S$ itself. So, we can use it to define an interpretation | | of the language. Any closed set of possibilities is an allowable interpretation of an atomic statement $p$. The constants $T$ and $\perp$ must be interpreted as $S$ and $\{\perp\}$, respectively, as $\top$ is true at every possibility and $\perp$ true only at $\perp$. This satisfies Sambin's general conditions on interpretations | |,

\footnotetext{
2 Sambin uses $\mathscr{F}$ for both the pre-topology and its closure operator; I'll use Rumfitt's notation $C$ for both.

3 "Saturated" in Sambin's terminology.
} 
according to which $|\mathrm{T}|=S=C(\mathrm{\top})$, and the condition mentioned above needed to verify explosion, $|\perp|=C(\emptyset)=\{\perp\}$. Contraction is valid since $x \bullet x=x$, and so $x \in C(x \bullet x)$.

The evaluation clauses for the connectives Rumfitt proposes are:

$$
\begin{aligned}
|A \wedge B| & =|A| \cap|B| \\
|A \vee B| & =C(|A| \cup|B|) \\
|A \rightarrow B| & =|A| \rightarrow_{C}|B| \\
|\neg A| & =|A|^{\perp}
\end{aligned}
$$

(C) and (D) agree with Sambin's definitions for $\&$ and $\oplus$, but Sambin does not give a clause for $\neg$. Instead, $\neg A$ is defined as $A \rightarrow \perp$, and we have an evaluation function $\rightarrow_{C}$ for the conditional. In Sambin, we have:

$$
|\neg A|=|A| \rightarrow_{C}|\perp|=|A| \rightarrow_{C}\{\perp\}
$$

Rumfitt's ${ }^{\perp}$ is the operation of orthocomplement, based on the notion of incompatibility: two possibilities are incompatible if $x \bullet y=\perp$. $U^{\perp}$ is the set of all possibilities incompatible with every possibility in $U$, i.e., $U^{\perp}=\{x: x \bullet y=\perp$ for all $y \in U\}$ (p. 167). The evaluation function for the "logician's conditional" is given by $U \rightarrow_{C} V=\{x: x \bullet y \in V$ for all $y \in U\}$ (p. 183). ${ }^{4}$ If we set $V=\{\perp\}$, the condition becomes $x \bullet y \in\{\perp\}$, i.e., $x \bullet y=\perp$, so the definitions of $|\neg A|$ agree.

\section{Negation}

The semantics of possibilities as a pre-topology is intuitively compelling, and Rumfitt ably shows how the evaluation rules for the connectives are forced upon us if we want to hold on to the usual inference rules being sound and complete. The critical assumption that makes the difference between intuitionistic and classical logic, however, is not one that is forced by an inference rule accepted on all sides: It is the condition that for any $A,\left(|A|^{\perp}\right)^{\perp}=|A|$. It is forced by double negation elimination, i.e., $\neg \neg A \Rightarrow A$, but validity of this rule is of course exactly at issue in the debate between intuitionistic and classical logic.

Not being able to appeal to an accepted inference rule to justify the condition characteristic of classical logic, Rumfitt needs a different strategy. It can easily be seen that $\left(\left(|B|^{\perp}\right)^{\perp}\right)^{\perp}=|B|^{\perp}$, for any $B$. So if it is the case that $|A|=|B|^{\perp}$, for some $B$, then we would have

$$
\left(|A|^{\perp}\right)^{\perp}=\left(\left(|B|^{\perp}\right)^{\perp}\right)^{\perp}=|B|^{\perp}=|A|^{\perp} .
$$

The crucial condition that $\left(|A|^{\perp}\right)^{\perp}=|A|$, and with it the validity of doublenegation elimination, comes down to this: is the set of possibilities that make $A$ true always the same as the set of possibilities that are incompatible with some other closed set of possibilities? The $A$ with this property are said to have a back: the back of $A$ is the set of those possibilities incompatible with $A$, and $|A|=|\neg B|$ iff

\footnotetext{
4 Sambin defines $U \rightarrow_{C} V=\{x: x \bullet y \in C(V)$ for all $y \in U\}$, and in our context we are only concerned with $V$ where $V=C(V)$.
} 
$|B|$ is the back of $A$. Putative examples of failures of double-negation elimination (and of the law of excluded middle) involve statements $A$ for which $|\neg A|$ is perhaps not the back of $A$ : then $|\neg \neg A| \neq|A|$ and $|A \vee \neg A| \neq S$.

Since possibilities are introduced as the truth grounds of possible statements, it seems the only way we can have evidence for the existence or non-existence of specific possibilities is as the truth grounds for possible statements. In other words, evidence that there is such a thing as the possibility that $p$ is the existence of a possible statement that would be true at that possibility. Supposing that for any statement $A$ its negation $\neg A$ is always a statement, there always is a possibility incompatible with the truth-grounds for $A$. If nothing else, the possibilities that together form the truth grounds for $\neg A$ all have this property, and at least $\perp$ is one such. But there may not be enough possibilities which are truth grounds of $\neg A$ to guarantee that $|\neg \neg A|=|A|$ and $|A \vee \neg A|=S$.

One way to justify that $|\neg A|$ is always large enough is to appeal to the intuitive validity of some rule of inference. This strategy, however, is not available. Any rule sufficiently strong to guarantee it, such as $\neg \neg A \Rightarrow A$, will be one the validity of which is rejected by those who deny the universal validity of non-intuitionistically valid inferences. This is a weakness built into the semantics of possibilities, since they only exist as truth-grounds for possible statements, and so we need prior evidence of the truth of a possible statement (e.g., that it is the conclusion of an intuitively accepted inference from true statements). Realist possible worlds semantics, e.g., does not face this problem. One may argue that certain (kinds of) possible worlds exist on grounds that do not depend on the truth or falsity of statements, or the intuitive validity of certain inference rules (say, maximality principles). A way to make this point vivid is this: $A$ has a back if there is some statement $B$ such that $A$ is equivalent to $\neg B$, i.e., $|A|=|\neg B|$ - then $|B|$ is the back of $A$. But there is no prima facie reason that every statement $A$ is equivalent to such a negation, other than perhaps to the negation of $\neg A$. But whether $A$ and $\neg \neg A$ are always equivalent is exactly what's at issue.

A dialectically weaker strategy is to examine putative examples of statements that do not have backs, i.e., where the set of possibilities incompatible with the truth grounds of $A$ do not form a closed set, and so are not all of the truth grounds of $\neg A$.

\section{Intuitionistic Mathematics}

The first such example considered by Rumfitt are so-called weak counterexamples in intuitionistic number theory. A weak counterexample (to the law of excluded middle) is a statement $A$ where $A \vee \neg A$ is not intuitionistically true. Suppose we have a decidable property of natural numbers, $P(n)$, e.g., "the sequence $s$ does not occur before the $n$-th place in the decimal expansion of $\pi$," or "if $n$ is even and $>2$, then $n$ is equal to the sum of two primes." 5 Then $\forall x P(x)$ is the statement that $s$ never appears in the decimal expansion of $\pi$, or that Goldbach's Conjecture is true, respectively. At present, we have neither a proof nor a refutation of either. Hence $\forall x P(x) \vee \neg \forall x P(x)$ is not intuitionistically true. In the context of truth-ground

\footnotetext{
5 Rumfitt uses Brouwer's original example of the occurrence of 0123456789 in the decimal extension of $\pi$. It is now known that it does occur (Borwein 1998), so assume $s$ is a sequence which so far has not been found among the decimal expansion of $\pi$.
} 
semantics, this means: we do not know if the set of truth-grounds for $\neg \forall x P(x)$ is the entirety of the possibilities incompatible with the truth grounds of $\forall x P(x)$, i.e., we do not know if $\forall x P(x)$ has a back. However, the question should be, Rumfitt urges, whether we should take that as evidence that $\forall x P(x)$ has no back, rather than, say, we simply don't yet know if it does.

The argument that we should not is roughly the following: since $P(n)$ is decidable, we are entitled to assert that $\forall x(P(x) \vee \neg P(x))$. So whether or not $\forall x P(x)$ or $\neg \forall x P(x)$ does not hinge on the question if there are $n$ such that the state of $P(n)$ is somehow indeterminate. It merely hinges on the questions of what the truth grounds for $\forall x P(x)$ are. The possibility that there is a proof of $\forall x P(x)$, say, from the axioms of Heyting arithmetic HA, is one truth-ground of $\forall x P(x)$. But could it be that $P(n)$ merely happens to be true for every natural number $n$, without this being provable from HA or otherwise "lie in the rule for" determining $P(n)$ ?

Rumfitt (p. 206ff) invites us to consider the result that ancestral logic-i.e., first-order logic augmented by an ancestral operator ${ }^{6}$ - suffices to categorically characterize the natural numbers. Once we have a categorical characterization of the natural number structure, we can reason from the determinacy of all instances $P(n)$ to the determinacy of quantified statements over the instances and get $\forall x P(x) \vee \exists x \neg P(x)$, from which the contested instance of excluded middle, $\forall x P(x) \vee \neg \forall x P(x)$ follows intuitionistically.

Rumfitt is of course right to say that the the intuitionist "will understand the explanation of the ancestral operator" (p. 207) and that "ancestral logic is part of the common ground [classical logicians] share with intuitionists" (p. 208). However, in order to make the crucial step from $\forall x(P(x) \vee \neg P(x))$ to $\forall x P(x) \vee \exists x \neg P(x)$, the intuitionist must also accept the result that ancestral logic provides a categorical characterization of the natural number structure. But it is not clear that he must. For the proof that ancestral logic does this requires classical reasoning about all models. Although I don't have a proof that no intuitionistically acceptable argument can be given, two reasons suggest that it is unlikely. The first reason is simply that the content of what would have to be proved would involve reasoning about models, i.e., quantification over completed infinite sets. The second is this: If the ancestral operator is strong enough to make quantification over the natural numbers determinate in the relevant sense, it would be expected that the mere addition of the ancestral operator to Heyting arithmetic would already result in a stronger system, as it does in the classical case. However, adding the ancestral to Heyting arithmetic is conservative (Arai 2010).

In Section 7.4, Rumfitt considers the case of smooth infinitesimal analysis (SIA) and concedes that classical logic cannot be applied within it. Here, the problem lies not in the indeterminacy of quantification (and hence the question whether, say, $\forall x P(x)$ has a back), but already in the indeterminacy of atomic statements. In SIA, the predicate "is identical to 0" has no back. One might think that sIA is an obscure theory, and that the failure of classical logic here is inextricably linked to the existence of nilsquares (quantities different from 0 whose square equals 0 ). However, the same kinds of counterexamples can be found in "ordinary" intuitionistic analysis, where identity (with 0 ) is likewise indeterminate, i.e., $\forall x(x=0 \vee \neg x=0)$ does not hold. But in intuitionistic analysis things are worse for the defender of classi-

6 The ancestral $R^{*}$ of a relation $R$ is the relation which holds between $x$ and $y$ iff there is a finite sequence $z_{i}$ such that $x R z_{1} R z_{2} \ldots z_{n} R y$. 
cal logic. As in SIA, we face examples of predicates without backs ( $x=0$ ") and corresponding failures of the law of excluded middle. Moreover, for some choices of $P(x)$, intuitionistic analysis not only fails to prove the corresponding instance of excluded middle, but proves its negations, i.e., $\neg \forall x(P(x) \vee \neg P(x))$ (e.g., when $P(x)$ is $x=0$, or Brouwer's own example, " $x$ is rational"). This makes it hard to see how Rumfitt's truth-ground semantics can be expanded to deal with intuitionistic analysis, even without accepting the principle that every statement (property) has a back or bringing in mysterios objects such as nilsquares.

\section{Vagueness}

In Chapter 8, Rumfitt takes on the challenge to classical logic arising from the consideration of vague predicates. Vague predicates, such as "is tall" or "is red," have often been taken to produce statements that violate excluded middle. When $a$ is borderline red, there is a strong intuitive inclination to deny, or at least withhold assent from, both $A$ and $\neg A$ (where $A$ is " $a$ is red"). Rumfitt puts forward another topological semantics which allows us to keep this intuition-deny bivalence for vague statements - and still yields classical logic.

In Rumfitt's semantics for polar predicates such as " $x$ is red," the extensions of predicates are regular open sets in a certain topology. Suppose, for instance, that we're considering 100 colored tubes, $a_{1}, \ldots, a_{100}$, each one indiscriminable from the next. Tube $a_{1}$ is red, and $a_{100}$ is orange. The predicate $R(x)$ is " $x$ is red" and the predicate $O(x)$ is " $x$ is orange." (The (shades of) the tubes $a_{1}$ and $a_{100}$ are the poles in this case.) The elements $U$ of the topology are the (shades of) the $a_{i}$. The interior $\operatorname{Int}(X)$ of a set $X \subseteq U$ is the set of those elements in the set that are maximally close in color to one of the poles (i.e., no other pole is at least as close), and a set is open if it is equal to its own interior. This defines a topology on $U$. The extension of a color predicate $R(x)$ is then given by the set of those $a_{i}$ that the red pole $a_{1}$ is maximally close to (in the sense that no other pole is as close), and the extension of $O(x)$ are those $a_{i}$ to which the orange pole $a_{100}$ is maximally close.

Extensions so defined are not just open sets, i.e., identical to their interior, but also identical to the interior of their closure. (In any topology, the closure of an open set is the complement of the interior of the complement: $\mathrm{Cl}(X)=$ $U-\operatorname{Int}(U-X)$.) Rumfitt shows that in the case of extensions of color predicates, this condition is satisfied, so extensions of color predicates are regular open sets in the topology on $U$. Again appealing to the validity of introduction and elimination rules for "and" and "or," he stipulates that the extension of the conjunction $\ulcorner A(x)$ and $B(x)\urcorner$ of two predicates must be the intersection of the extensions, and the extension of a disjunction $\ulcorner A(x)$ or $B(x)\urcorner$ must be the smallest regular open set containing both extensions, which is the interior of the closure of the union. If $X$ is the extension of $A(x)$, the extension of $\ulcorner\operatorname{not} A(x)\urcorner$ is $X^{\perp}=\operatorname{Int}(U-X)$. Rumfitt motivates this as required by the intuitive meaning of negation: the extension of $\ulcorner$ not $A(x)\urcorner$ consists of those $a_{i}$ "whose color status is incompatible with being $A . "$ This does not simply mean that they fail to satisfy $A(x)$, for then a borderline red tube, failing to be red, would lie in the extension of $\ulcorner\operatorname{not} R(x)\urcorner$. However, Rumfitt does not discuss the relevant notion of incompatibility in de- 
tail. ${ }^{7}$ We may, however, motivate the assignment of $X^{\perp}$ to not $A(x)$ on the basis of an accepted inference: $A(a)$, not $A(a) \Rightarrow \perp$. So certainly the extension of $A(x)$ and not $A(x)$ must be disjoint. And the largest regular open set disjoint from $X$ is $\operatorname{Int}(U-X)$. In the resulting semantics, it may happen that neither $R(x)$ nor not $R(x)$ applies to a given $a$ - as we may expect for a borderline red object $a$ and yet $\ulcorner R(x)$ or not $R(x)\urcorner$ applies to everything. For if $X$ is the extension of $R(x)$, then the extension of $\ulcorner R(x)$ or not $R(x)\urcorner$ is

$$
\operatorname{Int}(C l(X \cup \operatorname{Int}(U-X)))=U
$$

Although Rumfitt's semantics for vague predicates makes use of similar mathematical machinery as his truth-ground semantics, it bears pointing out that here we deal with a topology on the domain while there we considered a set of semantic values endowed with a pre-topology. In the present semantics for vagueness, every predicate either does or does not apply to an element of the domain. Specifically, it's not as if, for a borderline red $a, R(a)$ has some intermediate truth value in the semantics. For any $a$, either $a$ is in the extension of $R(x)$, or it is not. If it fails to be in the extension of $R(x)$, however, that does not guarantee that it is in the extension of not $R(x)$. But any assignment of extensions to predicates will induce an assignment of one of two truth values, $\mathrm{T}$ or $\mathrm{F}$, to every statement. In truth-ground semantics, however, the semantic value - the value assigned to a statement - is not either $\mathrm{T}$ or $\mathrm{F}$, but the set of its truth grounds. The failure of bivalence is explained by allowing both $R(x)$ and not $R(x)$ to fail to apply to $a$, rather than by assigning some non-true non-false value to $R(a)$. Other proposed semantics for vagueness, e.g., using degrees of truth, do assign non-true non-false values to $R(a)$. In fact, one such semantics assigns elements of a Boolean algebra to $R(a)$; then any non-top non-bottom element of the algebra represents a possible borderline case. Since the elements form a Boolean algebra, however, we still have classical logic (Weatherson 2004). ${ }^{8}$

Rumfitt's approach has much to recommend it. It takes into account that many vague predicates are what he calls polar. It makes precise the intuition that clear cases of red are surrounded by a "neighborhood" of red. It accounts for the intuition that every $a_{i}$ along the spectrum between red and orange should satisfy $\ulcorner R(x)$ or $O(x)\urcorner$, and provides a way in which we can explain that they also all satisfy $\ulcorner R(x)$ or not $R(x)\urcorner$ (including those $a_{i}$ that satisfy neither disjunct).

While the semantics is informative and appealing in the case of color poles and monadic predicates, the extension to multi-place predicate and its application to inference is less so. In order to accommodate inferences involving statement with terms for multiple objects, we should, according to Rumfitt, consider multiplace predicates in a new topological space. In order to deal with a statement like

\footnotetext{
7 He merely states: "[W]hich color predicates an object satisfies depends on which poles are maximally close in color to it. Hence, ... the members of $X^{\perp}$ will be those objects whose color status is incompatible with being $A "$ (p. 244).

8 Rumfitt's proposal for a semantics underwriting Wright's approach to the sorites using intuitionistic logic in Section 8.3 seems to also follow this pattern, as the appeal to Tarski's completeness result suggests that an interpretation maps statements to open sets in a topology. However, in the ensuing discussion, it seems that the open sets are, like in the semantics for polar predicates, extensions of the predicates and not possible values of statements. It is not obvious to me that we can this easily switch between a semantics of statements and a semantics of predicates and assume that the resulting logic is the same.
} 
$R(b) \wedge O(c)$ we should consider instead the two-place predicate $\ulcorner R(x)$ and $O(y)\urcorner$ and a topology on the set of pairs of colors, in which every pair of poles makes a new pole that is used to define the open sets of that new topology. So, $\langle b, c\rangle$ is in the extension of $\ulcorner R(x)$ and $O(y)\urcorner$ iff the pole $\left\langle a_{1}, a_{100}\right\rangle$ is maximally close to $\langle b, c\rangle$ (distance, presumably, taken pointwise). Rumfitt does not provide a detailed account of this topology, how it is generated from the original topology, and what the defining clauses for disjunction, conjunction, and negation amount to in the resulting topology. There are details that have to filled in, e.g., how the order and multiplicity of variables in a composite predicate determines which poles are considered in what order. There are some "sanity checks" that would have to be done, e.g., proofs that the semantics on the product topology agrees with the basic topology when all variables are identified. In any case, the intuitive appeal of the polar semantics in the case of monadic predicates is quickly lost when considering the product topology for multi-place relations.

There is a deeper worry, however. In order to evaluate an argument, we have to know how many poles there are - and the poles are now not just determined by the application (e.g., which basic color predicates are in play), but by how many singular terms are in play. The example Rumfitt considers involves 100 of them, and so there are $2^{100}$ poles in the product topology. If we add another color tube to the sorites sequence, we double the number of poles. But this is deeply unsatisfying: we have not changed the color predicates, we've just added one shade: to account for that single shade, we'd expect the elements of the topology to simply include this new shade with poles remaining fixed. But the product topology adds $100^{100}$ new elements!

This highlights a logical feature of the setup as well: When we analyze a complex argument such as the sorites, we can no longer consider each step in isolation. For even though a single step only involves two singular terms (e.g., $a_{i}$ and $a_{i+1}$ ), we must evaluate all of them in the complete product topology. This opens the possibility that a single step, valid when evaluated relative to a topology of pairs, is no longer valid when evaluated relative to a topology of 100-tuples.

If the resulting semantics saves classical logic in the context of vagueness, then it should explain what goes wrong in the sorites. Here's a slightly different version of the argument: The claim that there is no borderline between $a_{i}$ and $a_{i+1}$ can simply be stated as $A_{i}=\neg\left(R\left(a_{i}\right) \wedge \neg R\left(a_{i+1}\right)\right)$. So the vagueness of the color continuum between red and orange is expressed by the set or conjunction of all $A_{i}$ $(i=1, \ldots, 99)$. The $A_{i}$ together with $R\left(a_{1}\right)$ and $\neg R\left(a_{100}\right)$ are jointly inconsistent classically. I do not know if, in the power topology, all $A_{i}$ are true (i.e., every $\left\langle a_{i}, a_{i+1}\right\rangle$ is in the extension of the two-place predicate $\ulcorner\operatorname{not}(R(x)$ and not $R(y))\urcorner$. If they all are, the semantics does not save classical logic. If one of them is not, it seems according to the semantics we have a borderline case and so not succeeded in giving a semantic interpretation of vagueness.

\section{Conclusion}

Rumfitt's book puts forward a number of challenges that any opponent of classical logic has to grapple with. It also provides logicians with a wealth of promising ideas ripe for further development. The truth-ground semantics of possibilities could be certainly be expanded. How should we deal with quantifiers? How exactly does 
it deal with logical truth and consequence? What is the role of the "logician's conditional" and its relation to "if ...then"? Can it be adapted to serve the aims of those who reject some of the assumptions made? For instance, can it be made to apply to cases where statements can be both true and false at a possibility (other than $\perp$ ), or to accommodate purported counterexamples to explosion, cut, or weakening? The technical development of polar semantics for vague predicates is so far somewhat underdescribed. How does it deal with quantification? What is the relation between the statement operators $\neg, \vee, \wedge$ and the operators on predicates? Are we justified in moving between judgments about truth of statements and judgments of whether tuples of elements of the domain satisfy complex properties? Specifically, what is the relation of "not" applied to predicates and the "ᄀ" applied to statements?

Further development of the semantics sketched by Rumfitt promises to help clarify the underlying logical and semantic issues raised by challenges to classical logic. The picture I laid out in the introduction above suggests a further methodological task. It is one thing to give a semantics which, while validating classical logic, also provides an explanation for why, say, purported failures of excluded middle are not in fact failures. It is at this point that intuitive, and in some cases well-informed, judgments about such purported failures run up against the explanations provided by the proposed semantics. If there are good reasons why, say, we are inclined to deny " $a$ is red or $a$ is not red" in certain cases (e.g., evidence of the actual use of such judgments by English speakers), we must weigh the strength of these reasons against the persuasiveness of the proposed semantical explanation. The more complex the explanation, of course, the easier it is to reject it in the final analysis. One may reject it on technical grounds, say, if it does not validate a required inference. But one may also reject it as not being faithful to the data, and $\wedge, \vee, \neg$ as not correctly capturing the correspnding English expressions.

\section{References}

Arai T (2010) Intuitionistic fixed point theories over Heyting arithmetic. In: Feferman S, Sieg W (eds) Proofs, Categories and Computations: Essays in Honor of Grigori Mints, College Publications, pp 1-14

Borwein JM (1998) Brouwer-Heyting sequences converge. Mathematical Intelligencer 20(1):1415

Rumfitt I (2015) The Boundary Stones of Thought: An Essay in the Philosophy of Logic. Oxford University Press, Oxford

Sambin G (1995) Pretopologies and completeness proofs. Journal of Symbolic Logic 60(3):861878 , DOI $10.2307 / 2275761$

Weatherson B (2004) True, truer, truest. Philosophical Studies 123(1-2):47-70 